\title{
TV/Series
}

$17 \mid 2020$

Séries : les sens de l'Histoire

\section{Narcos et l'histoire dé-colombianisée de la Colombie}

\author{
Luis Rivera-Vélez
}

\section{(2) OpenEdition}

Journals

Édition électronique

URL : http://journals.openedition.org/tvseries/4253

DOI : 10.4000/tvseries.4253

ISSN : 2266-0909

\section{Éditeur}

GRIC - Groupe de recherche Identités et Cultures

\section{Référence électronique}

Luis Rivera-Vélez, « Narcos et l'histoire dé-colombianisée de la Colombie », TV/Series [En ligne], 17| 2020, mis en ligne le 24 juin 2020, consulté le 06 septembre 2020. URL : http://

journals.openedition.org/tvseries/4253; DOI : https://doi.org/10.4000/tvseries.4253

Ce document a été généré automatiquement le 6 septembre 2020

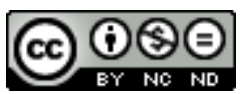

TV/Series est mis à disposition selon les termes de la licence Creative Commons Attribution - Pas d'Utilisation Commerciale - Pas de Modification 4.0 International. 


\title{
Narcos et l'histoire dé-colombianisée de la Colombie
}

\author{
Luis Rivera-Vélez
}

\section{Introduction}

1 En août 2015, Netflix sort la série Narcos (Netflix, 2015-201711) qui cherche à raconter « la vraie histoire de comment la cocaïne est devenue un tel problème aux États-Unis et en Europe, et comment tout a commencé à Medellín [Colombie] ${ }^{2} »$. Écrite par les scénaristes expérimentés Chris Brancato, Doug Miro et Carlo Bernard, et réalisée par José Padilha, Narcos bénéficie d'une très bonne réception et entre très vite dans le top 50 des meilleures séries télévisées selon le classement d'IMDb. Elle reçoit d'ailleurs de nombreuses récompenses ${ }^{3}$. De plus, la série bénéficie d'une stratégie publicitaire sans précédent $^{4}$, et même le président de la Colombie, Juan Manuel Santos, admet que la série est «bonne ». D'ailleurs le gouvernement colombien subventionne la production de la première saison ${ }^{5}$. Narcos est donc un succès pour Netflix et, positionnée comme une des meilleures productions de la plateforme d'après son directeur des programmes Ted Sarandos ${ }^{6}$, la série a réussi à s'ouvrir au marché hispanophone grâce à l'utilisation de l'Histoire colombienne ; une Histoire si tragique que parfois «la réalité dépasse la fiction » (selon le texte de l'incipit, S01E01).

2 Narcos s'inscrit dans un groupe de séries identifié par Benhessa et Bittinger ${ }^{7}$ qui abordent l'enjeu des drogues de manière originale. Alors que The Wire (2002-2008) est connue pour son approche sociologique d'un déterminisme social qui creuse les inégalités, et Breaking Bad (2008-2013) est connue pour la figure du self-made-man Walter

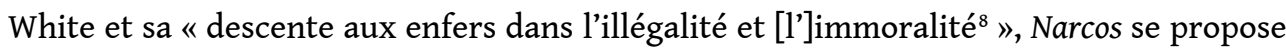
de dépasser la dichotomie agent/structure pour recréer un phénomène plus complexe en combinant les deux aspects : l'histoire du roi de la cocaïne dans un pays où l'absence d'opportunités fait du narcotrafic un phénomène social. Cette trilogie n'est pas exclusive car une série comme Weeds (2005-2012) aborde aussi la question du choix personnel d'une mère qui prend goût au business de la vente illégale de cannabis. Mais 
avec Narcos, Netflix inaugure une nouvelle ère des séries sur la drogue où l'accent est mis sur l'imbrication entre problèmes personnels et enjeux sociaux structurels, que ce soit dans des pays producteurs comme le Mexique avec la série El Chapo (2017-2018), ou dans des pays consommateurs comme aux États-Unis avec Ozark (2017-2019).

Par rapport aux autres séries qui abordent le sujet des drogues, la valeur ajoutée de Narcos est son fondement historique: la vie et l'œuvre de Pablo Escobar mises en images dans les deux premières saisons de la série. Profitant du caractère hors du commun de la biographie d'Escobar, Narcos propose un récit basé sur un canevas réaliste, assez commun dans les films et séries sur le trafic de drogue. Grâce à une production à grands moyens, la série permet aussi de populariser l'Histoire de la Colombie, rendant la frontière entre le savant et le populaire un peu plus étroite. L'adaptation est plutôt assumée comme une contrainte télévisuelle qui facilite la compréhension, que comme une source pour la fiction, en raison du nombre de personnages et du brouillage des frontières entre les clans qui se disputent le marché de la drogue en Colombie. La simplification de certains aspects historiques facilite la narration, ce qui est essentiel dans l'accroche de la série.

4 Toutefois, la réception colombienne de Narcos est ambiguë. À l'intérieur du pays, les critiques sont fortes et son succès très relatif : tant le gouvernement que la famille de Pablo Escobar, ainsi que les spécialistes de cinéma, questionnent l'image de puissance que la série véhicule du monde du trafic illicite de stupéfiants et notamment de cet anti-héros qu'est Pablo Escobar. Par exemple, après la sortie de la série, le fils d'Escobar dénonce les erreurs historiques et la mystification d'un Pablo Escobar «beaucoup plus gentil que dans la vraie vie ${ }^{9}$ ». De même, suite à la grande campagne publicitaire internationale qui a accompagné la série, le président de la Colombie, Juan M. Santos (2010-2018), a protesté contre l'exaltation d'Escobar comme « héros ${ }^{10}$ ». Au-delà de leur caractère politique, ces critiques semblent excessives du fait qu'elles négligent le caractère "fantastique ", à la fois amusant et tragique, de l'histoire de Pablo Escobar. De ce fait, sa vie répond facilement à un archétype cinématographique, tel un Tony ' Scarface' Montana. De plus, les incohérences historiques sont minimes, car les grandes lignes et personnages de l'histoire sont cohérents avec l'Histoire ${ }^{11}$.

5 La vie de Pablo Escobar est d'ailleurs devenue un sujet classique du cinéma ${ }^{12}$ et cela semble cohérent que ce succès soit aussi exploité par la télévision. Ainsi, il paraît important de signaler que la représentation de la vie des narcotrafiquants n'est pas en soi un problème pour les Colombiens. Narcos arrive sur les écrans trois ans après le feuilleton télévisé colombien Escobar, el patrón del mal (2012). Escobar a une narration moins complexe, plus longue (la version internationale compte 74 épisodes d'environ une heure, ce qui la rend plus difficile à regarder) et ne cherche pas à être attractive pour un public international. Toutefois, cette telenovela a captivé les Colombiens à tel point que son pilote est devenu le pilote le plus regardé de l'histoire de la Colombie. Par la suite, la série a été importée aux États-Unis par Telemundo, qui a fait d'elle la première production diffusée en prime time sans qu'elle soit une production originale. Sans compter le fait que Netflix a aussi acheté Escobar pour la diffuser sur sa plateforme à côté de Narcos.

6 Cependant, nous soutenons ici que le plus grand problème de la série vient de son manque d'engagement social, dans le sens où la forme du récit de Narcos est conservatrice et centrée sur les personnages étasuniens, ce qui ne permet pas de proposer un regard original sur l'histoire contemporaine de la Colombie, comme la 
série se propose de faire. Au contraire, à travers le récit étasunien de la vie d'Escobar, Narcos enlève son identité colombienne à l'histoire du narcotrafic, empêchant l'identification des colombiens à l'histoire qui est mise en scène, et donc la réflexion sociale sur le phénomène mis en scène. Nous défendons l'idée que Narcos échoue à séduire les Colombiens ${ }^{13}$, non pas par sa volonté de relater l'Histoire colombienne, mais par la manière dont elle raconte l'Histoire et lui donne une trame. Narcos donne une vision de l'histoire colombienne qui ne permet pas une identification de la part des Colombiens, alors que ce sont eux qui sont au centre de l'histoire.

7 Nous montrons ici que le support esthétique, conjugué à une vision du monde centré sur les États-Unis, rompent le processus d'identification de la part des Colombiens et enlèvent ainsi sa vraisemblance au récit proposé par Narcos. Alors que dès le premier épisode, l'objectif annoncé de la série est de montrer l'histoire de Pablo Escobar comme "l'homme qui secoua un pays jusqu'à ses fondements" (S01E01), Narcos montre davantage la vie des agents de la DEA en Colombie que celle des Colombiens. C'est donc ainsi que la série « dé-colombianise » l'Histoire de la Colombie. Pour ce faire, nous nous intéressons - de manière synthétique - au jeu des acteurs, au générique, à la photographie, aux canaux de financement, aux techniques filmiques, au scénario et aux traits psychologiques des personnages.

8 Après avoir exposé une courte réflexion théorique sur l'importance de la narration dans le processus d'identification, nous analyserons comment Narcos malmène l'Histoire de la Colombie tant sur la forme que dans le fond.

\section{La narration comme signe distinctif d'une série historique}

9 La narration des séries permet la fidélisation des téléspectateurs. Face à une narration complexe et sophistiquée, le format des "dramas», c'est-à-dire ces feuilletons où l'intrigue se déroule de façon séquentielle épisode par épisode, permet la création d'une intrigue qui s'approfondit et se démêle dans le temps, ce qui provoque une montée en puissance de l'histoire au fil des épisodes. La magie de la série est donc de permettre au spectateur l'identification avec les personnages grâce à la temporalité longue. Dans Narcos, le traitement de la temporalité est complexe et accrochant, notamment avec une première saison exposant plus de vingt ans de la vie d'Escobar, et une deuxième montre les derniers mois de celle-ci. Ainsi, si la narration permet la fidélisation à l'histoire, elle permet aussi l'adhésion aux personnages.

Les séries réalistes ont un canevas susceptible de créer plus d'identification et d'adhésion chez le spectateur, car leur représentation d'une situation sociale sert à «refaire société ", notamment en montrant un roman national qui deviendrait très positif quand il s'agit des minorités ou des populations subalterne ${ }^{14}$. Ainsi, par leur fort réalisme, les séries politiques contemporaines cherchent à provoquer un changement dans le roman national et permettre au spectateur de penser la réalité autrement, afin de valoriser des parties cachées de l'histoire, ces figures longtemps restées invisibles, qui gagnent une place et, ce faisant, permettent de proposer une lecture alternative de l'histoire, des visions humanisées et romantiques de la politique et du monde qui les entoure. 
11 L'engagement politique des séries peut être leur force. The Wire, par exemple, est « une critique puissante du néolibéralisme et de ses conséquences sociales ${ }^{15}$ ", notamment par l'étude des effets des politiques urbaines qui construisent une séparation entre quartiers gentrifiés et quartiers ghettoïsés. Pour les séries historiques, l'engagement pourrait permettre une meilleure compréhension du passé afin de mieux anticiper le futur. C'est (peut-être) un défi plus complexe mais aussi plus provocant.

Dans la série The Crown (2016) de Netflix, le réalisme joue un rôle crucial tant sur l'esthétique (le budget de la série est un des plus élevés de l'histoire de la télévision ${ }^{16}$ ) que sur le fond historique. Créée et écrite par Peter Morgan, scénariste du film The Queen (2006) et auteur de la pièce de théâtre The Audience (2013), la série a reçu de nombreux prix, et la critique l'a flattée tant sur le fond, assez fidèle sur les faits historiques, que sur la forme, avec des acteurs captivants ${ }^{17}$. Mais une des particularités de The Crown est de vouloir proposer un traitement alternatif de « l'histoire derrière les façades des deux des adresses les plus importantes du monde - le palais de Buckingham et 10 Downing Street - avec toutes les intrigues, les histoires d'amour et les machinations derrière les plus grands événements du $20^{\mathrm{e}}$ siècle ${ }^{18}$. Derrière l'histoire de famille (royale certes), le but est de proposer une lecture « d'un empire en déclin, un monde en désordre et l'aube d'une nouvelle ère ${ }^{19} »$. L'histoire de la famille royale britannique s'inscrit donc dans celle d'une société, voire d'un monde en évolution.

Dans Narcos, cet objectif semble plus flou. Selon le directeur José Padilha, la volonté explicative du récit est de retracer l'histoire du trafic des drogues, notamment dans ses liens avec les États-Unis et l'Europe ${ }^{20}$. Or, l'ensemble de la série se déroule en Colombie, et les personnages étatsuniens ne se soucient que de la façon dont les États-Unis contribuent à façonner l'histoire colombienne. En conséquence, les spectateurs étasuniens ne peuvent s'identifier qu'à des personnages immergés dans un autre pays (la Colombie), et les Colombiens n'ont comme repère que ceux que la série cherche à dénoncer : les narcotrafiquants et les hommes politiques corrompus ou incompétents.

La série introduit ainsi une vision caricaturale des personnages, faisant des Colombiens les méchants d'une histoire où les victimes sont les gringos étasuniens. Pour résumer à grands traits, les Américains viennent sauver la Colombie du fléau des drogues, car les Colombiens sont incapables de le faire eux-mêmes.

15 Ceci pose un problème quant à l'enjeu de produire des séries fictives fondées sur l'Histoire. Par définition, la fiction a des libertés de mise en scène que le documentaire n'a pas. Il est donc important de traiter Narcos comme une fiction, afin d'éviter que la responsabilité de la différenciation entre la réalité et la fiction retombe sur le spectateur au moment du visionnage ${ }^{21}$. Un principe de précaution exige donc que, pour ne pas avoir une idée fausse de la réalité, il est nécessaire de partir du constat que tout ce qui est diffusé dans une série est une fiction pour divertir. Or, quand Netflix présente sa série comme étant « inspirée de faits réels " mais comportant des "éléments fictifs dont toute similitude avec des personnes existantes serait fortuite" (S01E01) les frontières entre l'Histoire, le réalisme et la fiction sont brouillées. Si l'on considère la série comme du "réalisme historique ", on accepte que les faits exposés aient eu lieu (au moins pour ce qui est des événements importants). À l'inverse, si l'on considère la série comme une "fiction historique ", alors il est nécessaire de se détacher du détail des événements et de plutôt considérer la véracité du contexte où se déroule l'action.

Narcos prend le parti de représenter des faits historiques de la manière la plus fidèle possible, ce qui montre son intention d'intégrer la catégorie du réalisme historique. Or, 
étant centrée sur des acteurs américains et ayant utilisé des acteurs non-Colombiens pour jouer des personnages locaux, la série donne l'impression d'une réinvention de l'Histoire, ou dans le meilleur des cas, d'un récit inachevé et incomplet sur l'histoire du trafic de drogue en Colombie. Même si l'on considère que l'objectif de la série est plutôt de divertir que d'informer, le récit proposé tout au long de Narcos semble invraisemblable pour les Colombiens. Un critique de télévision colombien, Ómar Rincón, va même jusqu'à affirmer que «Narcos est la vision de Miami et des États-Unis de NarColombie, quelque chose comme l'idée qu'a Trump [des Colombiens] : les bons sont les gringos formés à la DEA. Et les Narcos sont inadaptés comiques ou des primitifs de mauvais goût ${ }^{22}$ ». Sans en arriver à de telles affirmations, nous tâchons de montrer ici comment Narcos échoue à représenter le phénomène social du narcotrafic en Colombie.

\section{Une forme décolombianisée de Pablo Escobar}

17 Narcos fait le pari de faire vivre l'Histoire de la Colombie aux téléspectateurs du monde entier. Quand le gouvernement finance la production de la première saison, le but est double: dynamiser l'économie nationale à travers l'impulsion de l'industrie de l'audiovisuel colombienne et profiter de la plateforme pour «exposer au niveau international le talent et les paysages de la Colombie ${ }^{23}$ ». Un Colombien exigeant ferait très certainement la critique que l'objectif du gouvernement n'a (littéralement) pas été respecté car certaines scènes confondent Medellin et Bogota (e.g. à la minute 17 de l'épisode S01E08, la plaine de Bogota est repérable alors que les acteurs devraient être entre les montagnes de Medellín), mais en réalité l'ensemble de la production de Narcos est réussi. D'un côté, les costumes et les décors sont très cohérents avec l'époque où l'histoire a lieu. La vraisemblance visuelle a été une des plus grandes exigences de la production, et l'anecdote la plus fameuse de la série affirme que la scène de la mort d'Escobar (S02E10) a été tournée sur le toit même où le vrai Escobar a été tuééc.

D'un autre côté, la technique cinématographique est très bonne. Des prises de vue aériennes, des plans très sensibles aux émotions et une fluidité de mouvement de la caméra font de la série une grande production ${ }^{25}$. De même, le générique de la série est très original et captivant. Si l'on pense le générique d'une série comme sa "fiche d'identité ${ }^{26}$, Narcos est sensuelle (musique ${ }^{27}$ ) et réaliste (utilisation d'archives) : le rythme de la série est soutenu, et les fondements historiques échappent à toute critique, grâce au pari du réalisme que la série propose. En somme, Narcos cherche à rendre hommage à la Colombie en retraçant son histoire de la manière la plus fidèle permise par une histoire dramatique.

Toutefois, malgré l'énorme budget de Narcos, un défaut est impardonnable pour un Colombien : le choix des acteurs ${ }^{28}$. Escobar est joué par Wagner Moura, brésilien ayant une très bonne relation avec le directeur de la série José Padilha. En effet, Moura et Padilha ont travaillé au préalable ensemble, dans un film sur les drogues à Rio de Janeiro (Troupe d'élite, 2007) qui est un succès dans les salles brésiliennes (2 371734 entrées selon le site adorocinema ${ }^{29}$ ) et gagne l'Ours d'Or de la Berlinale en 2008. Ainsi, pour Netflix c'est ce travail qui assure le poste à Moura. Dans un communiqué de presse, le responsable des contenus de Netflix, Ted Sarandos, affirme que José Padilha et Wagner Moura « ont créé un des portraits de la criminalité et la corruption policière 
plus sophistiqués et effrayants » et donc que Narcos est pensé «like nothing ever seen before ${ }^{30} »$.

Or, avant de commencer le tournage Moura ne parlait pas espagnol et sa corpulence n'était pas assez proche de celle de Pablo Escobar. Pour l'acteur lui-même, il s'agit d'une erreur de casting: "This is a big case of miscasting. One of the biggest miscasting in the history of [television]: I was super skinny - 40 pounds lighter - and I didn't speak a word of Spanish $»^{31}$. Netflix ne savait pas si la série allait être totalement en anglais ou bilingue anglais-espagnol, donc quand la décision est prise trois mois avant le début du tournage, Moura part à Medellín pour apprendre l'espagnol dans une université pour étrangers ${ }^{32}$.

Une grande différence d'accent est perceptible entre les deux saisons où Moura joue, et évidemment le jeu de Moura est plus crédible à la deuxième saison. Mais, deux aspects aggravent ce «miscasting»: Moura n'est pas le seul acteur qui a un fort accent noncolombien (voire de Medellín, qui est encore plus difficile à maîtriser) et, surtout, audelà de l'accent, c'est le monde des narcotrafiquants qui est mal représenté. Ainsi, l'actrice qui joue la mère de Pablo, Doña Hermilda (Paulina García) est chilienne et ne parle pas comme le ferait une femme de Medellín (S01E02). Ces personnages clés font tâche - pour un Colombien - quand on les compare à ceux de Gustavo Gaviria (Juan Pablo Raba), Judy Moncada (Cristina Umaña) ou Don Berna (Mauricio Cujar), tous Colombiens et qui jouent dans la série. Or, même des acteurs non-colombiens ont su représenter par leur accent et leur incarnation d'une culture très particulière, comme le fait l'interprète de la femme de Pablo Escobar, Tata (Paulina Gaitán) qui est Mexicaine. Ce problème est d'autant plus frappant quand on le compare aux personnages étasuniens, tous représentés par des acteurs natifs.

L'accent n'est pas le seul souci du choix de l'acteur. Si l'on excepte l'espagnol mal parlé que même les sous-titres corrigent (e.g. S01E05), le problème est que les acteurs doivent interpréter des personnages ancrés dans une culture qui permet une identification du spectateur à la scène qu'il est en train de voir. Même un très bon script a donc du mal à faire ressentir l'argot des gangs de Medellín et, sans cette fidélité culturelle, la série perd toute sa crédibilité pour le public colombien. Pour donner un contre-exemple, et en restant dans les productions historico-réalistes de Netflix, il serait impossible d'imaginer la Reine Elisabeth II dans The Crown jouée par une femme à l'accent indien, au lieu de la très anglaise Claire Foy.

Cet aspect de Narcos est très négatif quand il est comparé à la telenovela Escobar, el patrón del mal (Caracol Televisión, 2012). L'interprétation d'Andrés Parra comme Pablo Escobar a été tellement réussie que même les personnes proches d'Escobar ont salué le jeu de l'acteur. Si l'on en croit ce dernier, il a été sollicité par Netflix pour jouer dans Narcos, mais son succès était tel qu'il ne voulait pas rester étiqueté comme Escobar tout le reste de sa carrière ${ }^{33}$.

24 Comme on l'a dit plus haut, la deuxième saison améliore l'esthétique narrative de la série car Moura parle un meilleur espagnol et incarne plus subtilement Escobar, la relation de Peña et Murphy devient une amitié de confidence, et l'ensemble des personnages grandissent et sont plus solides, ce qui facilite l'identification. Représentant les derniers mois d'Escobar, et non pas 20 ans comme la première saison, l'histoire racontée pendant les dix épisodes de la deuxième saison est celle de l'effondrement physique et émotionnel d'Escobar, de l'empowerment de sa femme, Tata, pour sauver ses enfants, de la douleur d'une mère quand on tue son fils. 
La narration fait que la série soit plus dynamique, voire qu'elle avance plus vite, alors que la temporalité de l'intrigue est plus courte. Mais l'histoire des alliances est difficile à suivre, les tournures de la trame deviennent complexes et les émotions sont plus fortes. Ainsi, la deuxième saison se fait plus attractive car il y a plus de désespoir, plus d'enjeux, et le jeu politique est plus sordide. Mais sur le fond cependant, la relation entre les États-Unis et la Colombie ne change pas.

\section{Narcos, ou une histoire des États-Unis en Colombie}

La « guerre contre la drogue » a été une croisade morale étasunienne qui s'est répandue partout dans le monde à travers une diplomatie politique ${ }^{34}$, mais aussi via un terreau prohibitionniste favorable dans tous les pays (la consommation des drogues est vue comme problématique partout). De cette vision de l'enjeu, la dichotomie entre les États-Unis et les « autres » devient évidente : il y a ceux qui appuient la politique et ce qui ne font rien, et ceux qui mettent donc en danger la sécurité des États-Unis.

Dans Narcos, les agents de la DEA se présentent comme les défenseurs de cet intérêt américain face à deux ennemis: les trafiquants illégaux et la police colombienne corrompue. La conjoncture colombienne de l'époque est réaliste, mais l'importance que se donnent les deux agents de la DEA dans la série est plus éloignée de la réalité et rend la division États-Unis/Colombie caricaturale, créant une frontière entre "bons " et "méchants » selon si les personnages sont colombiens ou pas. La vraisemblance de la fiction est par conséquent remise en cause et ainsi, la capacité de la série à s'inscrire dans un débat plus ample sur la société et les phénomènes qu'elle met à l'écran.

Si l'ont fait une typologie des personnages représentés lors des premiers épisodes de la série, on peut trouver deux types de Colombiens et un type d'Américain. Premièrement, il y a les narcotrafiquants qui vivent le "Colombian dream»: ils sont assassins, délinquants et ont du succès (S01E03). Deuxièmement, il y a les Colombiens stupides (comme l'officier de l'aéroport dans l'épisode S01E02) qui sont corrompus, délinquants et se laissent acheter par un peu d'argent. Troisièmement, il y a les gringos incorruptibles et sauveurs, qui protègent les hommes politiques (S01E03 et S01E06) et qui vont jusqu'à prétendre utiliser une facture pour se faire rembourser un pot-de-vin (S01E03). Cette dichotomie devient très explicite quand dans l'épisode 2 de la première saison ("L'épée de Simon Bolivar») se succèdent deux scènes de sexe : une où Pablo trompe sa femme avec une journaliste et finit par la sodomiser de manière agressive (18'00-20'05), et une autre où Peña couche avec une prostituée de manière si sensuelle et attentive qu'à la fin cette dernière le remercie et lui lance un "tu es incroyable " (20'05-23'28). La brutalité d'un personnage s'oppose ainsi, et de manière très directe, à la douceur de l'autre.

Les acteurs latino-américains de Narcos réfutent cette critique parce qu'ils croient qu'il est difficile de classer comme héros ou méchants les personnages de la série ${ }^{35}$. Tel un Tony Soprano, Pablo Escobar est un père aimant et un assassin sans scrupules. Le colonel Carrillo est un patriote et un tortionnaire. Le président Gaviria est modéré mais stupide. Les agents de la DEA sont engagés et complices. Or, c'est autour de ces derniers personnages que l'ambigüité entre la narration focalisée sur la complexité des personnages et la division du monde entre les bons et les méchants se cristallise. 
30 Le neuvième épisode de la première saison («La Catedral ») est emblématique du discours narratif qui caractérise Narcos. Alors que Murphy et sa femme vont acheter des fleurs et discutent du prénom à donner à leur fille adoptive, un incident dans la rue trouble l'agent et le pousse à réagir violemment, allant même jusqu'à tirer sur la voiture qu'il a précédemment frappée (minutes 5'50 - 8'28). Murphy devient donc violent comme réponse à la manière d'agir des trafiquants illégaux. Or, dans le même épisode, Pablo Escobar assassine très violemment ses collaborateurs les plus proches, provocant le bain de sang le plus explicite de la saison (40'10-43'20). Face à cette violence, les gringos redeviennent sensés et décident de revenir à la légalité en s'adressant à la presse avec leurs preuves pour exposer Escobar face à l'inaction du gouvernement (46’50-50'09). Le cadre des scènes colombiennes est ainsi tâché de sang et violence, alors que celui montrant les personnages étasuniens met en scène la douceur des fleurs et d'un bébé.

31 Les scènes comme celle-ci sont très courantes tout au long de la série. Les Colombiens sont présentés comme étant stupides (le président qui accepte l'accord d'Escobar sur une prison contrôlée par lui-même) ou barbares (les violences des narcotrafiquants ou la police). Or, quand les Étatsuniens sont confrontés à ces violences, ils récupèrent leur intégrité et s'opposent à la sauvagerie (S02E03). En somme, les USA sont présentés comme victimes de la Colombie, soit parce que les drogues sont en train de détruire la société (S01E01), soit parce que le contexte de violence en Colombie les pousse à perdre leur humanité (S01E08). Cette manière de se raconter est de la pure mauvaise-foi sartrienne, où la conviction de privation de liberté (un contexte de violence généralisée) permet de justifier des faits pas toujours éthiques ${ }^{36}$.

32 L'approche centrée sur l'expérience des Américains en Colombie dérive d'une proximité de ces deux agents avec la production de la série. Les vrais agents Peña et Murphy ont été embauchés comme consultants pour $\operatorname{Narcos}^{37}$ et les acteurs Pedro Pascal et Boyd Holbrook ont reçu une formation avec ceux qu'ils incarnent ${ }^{38}$. La vision de l'histoire de la Colombie que Narcos véhicule le point de vue des agents du gouvernement étasunien, et par conséquence rend difficile une identification avec les personnages centraux, notamment si l'on prend en compte le public jeune, urbain et éduqué de Netflix.

33 Alors que la voix-off joue un rôle important en faveur de la généralisation d'une histoire personnelle, le narrateur de Narcos, Murphy, est emphatique et rend difficile l'adhésion à sa cause, voir impossible l'identification des Colombiens aux Étatsuniens (gringos). La narration est centrée sur la biographie des personnages, et non pas comme une explication des dynamiques collectives et les enjeux profonds liés à la construction démocratique de la Colombie à ce moment précis du développement du pays. Par conséquent, le réalisme cherché par la série entre en contradiction avec une réflexion introspective que le spectateur ferait de l'Histoire en regardant la série. Le message politique de Narcos est donc affaibli car la réflexion historique critique devient difficile et la série ne développe pas une lecture alternative des faits.

34 La série sert à véhiculer une politique spécifique (la « guerre contre la drogue »), qui continue à être prônée par les États-Unis (notamment par des institutions comme la DEA) et qui a des conséquences sociales néfastes. Les vrais agents Peña et Murphy continuent à prôner les recettes d'il y a trois décennies pour la lutte contre le narcotrafic au Mexique ${ }^{39}$, alors que même les acteurs de Narcos trouvent ces visions 
rétrogrades ${ }^{40}$. L'objectif de montrer une nouvelle facette de l'histoire du trafic de drogues échoue donc.

\section{Conclusion}

Narcos est une série esthétiquement réussie et fondée sur des faits historiques, bénéficiant d'une campagne publicitaire publicité «à la Netflix » (dans le métro et la rue des capitales européennes) qui la rend attractive, mais en même temps malaimée des Colombiens. Au-delà de quelques maladresses de communication, comme l'affirmation que "Pablo est la victime de la deuxième saison " $^{41}$, nous affirmons ici que sa mauvaise réception en Colombie répond à deux caractéristiques: le choix d'un acteur qui n'était pas le mieux placé pour incarner Pablo Escobar, et une histoire autour de deux agents américains.

Parce que (1) la société que la série cherche à représenter ne permet pas une identification de la part du spectateur colombien en raison du caractère "exotique " des acteurs, (2) le point de vue de la narration lui est étranger, et, (3) la réflexion sur le temps présent s'éloigne trop de la réalité nationale. L'esthétique narrative de Narcos décolombianise donc l'Histoire de la Colombie, et en ce faisant permet de poser la question de l'utilité et pertinence de l'usage d'une histoire nationale (celle de la Colombie) dans un but économique (celui de Netflix).

Le but affiché de Narcos est d'expliquer l'origine de narcotrafic, ce qui servirait à penser la politique de « guerre contre la drogue » que tous les pays du monde ont mis en place. Or, la série ne le fait pas. Elle aurait pu proposer une approche originale de l'histoire, ou montrer une réalité alternative et fictionnelle, telle que la série se propose de faire. Mais l'opportunité de proposer une lecture alternative de l'histoire colombienne n'a pas été saisie, et la série ne réussit pas à montrer pourquoi la vie d'Escobar importe aujourd'hui. Au contraire, Narcos continue à véhiculer l'image d'une intervention étatsunienne nécessaire pour résoudre les problèmes latino-américains, malgré des points de vue critiques sur la politique de prohibition des drogues, qui existent depuis les années 1990 et dont la série rend compte (S01E07). Cette même vision est véhiculée dans la saison 3 de Narcos qui montre le développement du marché illicite de drogue en Amérique latine après la mort de Pablo Escobar. Si le récit reste le même, le seul effet de la série sera de produire " plata » pour Netflix et « plomo » pour les pays qui vivent la guerre contre les drogues ${ }^{42}$.

\section{BIBLIOGRAPHIE}

BACQUÉ Marie-Hélène, FLAMAND Amélie, PAQUET-DEYRIS Anne-Marie et TALPIN Julien (éd.), The Wire. L'Amérique sur écoute, Paris, La Découverte, 2014.

BENHESSA Ghislain et BITTINGER Nathalie, «Poudre aux yeux. Autour de The Wire, Breaking Bad et Narcos », Esprit, n²2, 2017, p. 100-106. 
BRITTO Lina, « Car Bombing Drug War History », Nacla Report on the Americas, vol. 48, n²2, p. $177-180$.

BRODZINSKY Sibylla, « Narcos is a hit for Netflix but iffy accents grate on Colombian ears ", The Guardian (Royaume-Uni), 17 septembre 2015, consulté le 20 février 2019 : https:// www.theguardian.com/world/2015/sep/17/narcos-netflix-colombian-accents.

CHAPARRO Luis, « Los agentes de la DEA que capturaron a Pablo Escobar nos dicen cómo atrapar al Chapo », Vice (Amérique latine), 3 novembre 2015, consulté le 20 février 2019 : https:// www.vice.com/es_latam/article/pp5kdg/los-agentes-de-la-dea-que-capturaron-a-pablo-escobarnos-dicen-como-atrapar-al-chapo.

CUÉ Carlos, « 'Mi padre era mucho más cruel que el Pablo Escobar de Netflix », El País (Espagne), 28 septembre 2016, consulté le 20 février 2019 : https://elpais.com/internacional/2016/09/23/ argentina/1474656834_899105.html.

FAURE Antoine et TAÏEB Emmanuel, « Temporalité de la politique alternative dans les séries ». Quaderni, vol. 86, nำ 1, 2015, p. 23-37.

FAURE Antoine et TAÏEB Emmanuel, « Les 'esthétiques narratives' : l'autre réel des séries ». Quaderni, vol. 88, n³, 2015, p. 5-20.

FULLERTON Laurie, « How Netflix is growing the popularity of Narcos through smart social media marketing», The Drum, 6 septembre 2016, consulté le 20 février 2019 : https://

www.thedrum.com/news/2016/09/06/how-netflix-growing-popularity-narcos-through-smartsocial-media-marketing.

JOST François, « Fiction historique et réalisme », entretien publié par le Conseil Supérieur de l'audiovisuel, consulté le 20 février 2019 : https://clesdelaudiovisuel.fr/Analyser/Medias-etrealite/Fiction-historique-et-realisme.

KEETON Joey, « Why the bad first season of 'Narcos' is worth watching », The Daily Dot (États-Unis), 5 septembre 2015, consulté le 20 février 2019 : https://www.dailydot.com/upstream/narcosnetflix-original-first-season-review/.

LOUGHREY Clarisse, " The Crown: The Netflix show is the most expensive TV series ever ", Independent (Royaume-Uni), 2 novembre 2016, consulté le 20 février 2019 : https:// www.independent.co.uk/arts-entertainment/tv/news/the-crown-netflix-most-expensive-showever-matt-smith-a7392911.html.

MCALLISTER William, Drug diplomacy in the twentieth century: An international history. Londres/New York, Oxford University Press, 2000.

MANGAN Lucy, "The Crown review - the $£ 100 \mathrm{~m}$ gamble on the Queen pays off royally ", The Guardian (Royaume-Uni), 4 novembre 2016, consulté le 20 février 2019 : https:// www.theguardian.com/tv-and-radio/2016/nov/04/the-crown-review-netflix-100m-gamble-onthe-queen-pays-off-royally.

MORAES Camila, « ¿Con qué Pablo Escobar te quedas? 'Narcos' de Netflix es tan solo uno de los productos audiovisuales sobre el narcotraficante », El País (Espagne), le 7 septembre 2015, consulté le 20 février 2019 : https://elpais.com/cultura/2015/09/04/actualidad/ 1441389093_610905.html.

MORENO Carolina, « Netflix's 'Narcos' Series on Pablo Escobar 'Will be like nothing ever seen before' », Huffington Post (Mexique), 3 avril 2014, consulté le 20 février 2019 : https:// www.huffingtonpost.com.mx/entry/netflix-narcos-_n_5087063. 
MUSTO David, The American Disease: Origins of Narcotic Control, Oxford, Oxford University Press, 1999.

RINCÓN Omar, « Amamos a Pablo, odiamos a los políticos », Nueva Sociedad, janvier-février 2015, p. 94-105.

RINCÓN Omar, « Narcos da risa /El otro lado », El Tiempo (Colombie), 6 septembre 2015, consulté le 20 février 2019 : https://www.eltiempo.com/archivo/documento/CMS-16346263.

SARTRE Jean-Paul, L'Etre et le néant, Paris, Gallimard, 1976.

SHEPERD Jack, « Netflix claims Narcos is more popular than Game of Thrones », Independent (Royaume-Uni), 8 décembre 2015, consulté le 20 février 2019 : https://www.independent.co.uk/ arts-entertainment/tv/news/netflix-claims-narcos-is-more-popular-than-game-of-thronesa6764381.html.

SUAREZ SANG Lucia, « Ex-DEA agents who fought Pablo Escobar headline new NatGeo documentary ", Fox News (États-Unis), 30 août 2016, consulté le 20 février 2019 : https:// www.foxnews.com/entertainment/ex-dea-agents-who-fought-pablo-escobar-headline-newnatgeo-documentary.

TESTE Camille, « Que pensent les Colombiens de la série 'Narcos' ? », Les Inrockuptibles, 26 février 2018, consulté le 20 février 2019 : https://www.lesinrocks.com/2018/02/26/series/series/quepensent-les-colombiens-de-la-serie-narcos/.

\section{NOTES}

1. Nous distinguons ici les deux premières saisons (2015 et 2016) de la troisième (2017) parce que l'article se concentre sur la représentation de la vie de Pablo Escobar. Par ailleurs, il est important de différencier les séries Narcos, se centrant sur la Colombie, de Narcos: Mexico (2018), cette dernière devant être la quatrième saison de Narcos, mais se développant comme une série indépendante écrite et produite par Doug Miro et Carlo Bernard.

2. Carolina Moreno, «Netflix's 'Narcos' Series on Pablo Escobar 'Will be like nothing ever seen before'», Hiffington Post (Mexique), 3 avril 2014, consulté le 20 février 2019: https:// www.huffingtonpost.com.mx/entry/netflix-narcos-_n_5087063.

3. Voir la liste complète sur le site Wikipédia, consulté le 20 février 2019: https:// en.wikipedia.org/wiki/Narcos.

4. Des nombreux articles de presse peuvent témoigner de l'innovation publicitaire de Narcos, la considérant de «smart social media marketing " (Laurie Fullerton, « How Netflix is growing the popularity of Narcos through smart social media marketing», The Drum, 6 septembre 2016, consulté le 20 février 2019 : https://www.thedrum.com/news/2016/09/06/how-netflix-growingpopularity-narcos-through-smart-social-media-marketing), «massive » et envahissante (« Quand la série Narcos envahit le métro parisien », Creapills, 21 septembre 2016, consulté le 20 février 2019 : https://creapills.com/narcos-metro-paris-netflix-20160921), voire " géniale » ( La géniale opération marketing de Narcos qui laissera des traces ", Llllitl, 31 août 2017, consulté le 20 février 2019: https://www.llllitl.fr/2017/08/netflix-narcos-cocaine/). Le directeur de marketing de Netflix France, Benjamin Cléry-Melin, annonce même leur capacité à «améliorer considérablement la performance " grâce à l'innovation de la campagne publicitaire autour de Narcos (« Netflix - Narcos S1», Biborg, consulté le 20 février 2019: https://www.biborg.com/ work/netflix-narcos-s1). 
5. «Presidente Santos se reune con Netflix », article publié par le ministère de la culture de la Colombie, consulté le 20 février 2019 : http://www.mincultura.gov.co/prensa/noticias/Paginas/Presidente-Santos-se-reúne-con-Netflix.aspx.

6. Jack Sheperd, "Netflix claims Narcos is more popular than Game of Thrones ", Independent (Royaume-Uni), 8 décembre 2015, consulté le 20 février 2019: https://www.independent.co.uk/ arts-entertainment/tv/news/netflix-claims-narcos-is-more-popular-than-game-of-thronesa6764381.html.

7. Ghislain Benhessa et Nathalie Bittinger, « Poudre aux yeux. Autour de The Wire, Breaking Bad et Narcos ", Esprit, no2, 2017, p. 100-106.

8. Ibid., p. 103.

9. Carlos Cué, « Mi padre era mucho más cruel que el Pablo Escobar de Netflix », El País (Espagne), 28 septembre 2016, consulté le 20 février 2019 : https://elpais.com/internacional/2016/09/23/ argentina/1474656834_899105.html.

10. «Santos se refiere a serie 'Narcos' : ‘Escobar no merece ser exaltado' », El Tiempo (Colombie), 14 décembre 2016, consulté le 20 février 2019: https://www.eltiempo.com/politica/gobierno/ presidente-juan-manuel-santos-se-refiere-a-la-serie-narcos-41576.

11. Pour une étude approfondie des imprécisions historiques voir l'analyse de Lina Britto, "Car Bombing Drug War History ", Nacla Report on the Americas, vol. 48, n², p. 177-180.

12. Camila Moraes, «¿Con qué Pablo Escobar te quedas? ‘Narcos’ de Netflix es tan solo uno de los productos audiovisuales sobre el narcotraficante», El País (Espagne), le 7 septembre 2015, consulté le 20 février 2019: https://elpais.com/cultura/2015/09/04/actualidad/ 1441389093_610905.html.

13. Camille Teste, "Que pensent les Colombiens de la série 'Narcos'? ", Les Inrockuptibles, 26 février 2018, consulté le 20 février 2019 : https://www.lesinrocks.com/2018/02/26/series/series/ que-pensent-les-colombiens-de-la-serie-narcos/.

14. Antoine Faure et Emmanuel Taïeb, «Temporalité de la politique alternative dans les séries ». Quaderni, vol. 86, nº 1, 2015, p. 23-37 https://journals.openedition.org/quaderni/861.

15. Marie-Hélène Bacqué, Amélie Flamand, Anne-Marie Paquet-Deyris et Julien Talpin (éd.), The Wire. L'Amérique sur écoute, Paris, La Découverte, 2014, p. 10.

16. Clarisse Loughrey, "The Crown: The Netflix show is the most expensive TV series ever ", Independent (Royaume-Uni), 2 novembre 2016, consulté le 20 février 2019: https:// www.independent.co.uk/arts-entertainment/tv/news/the-crown-netflix-most-expensive-showever-matt-smith-a7392911.html.

17. Lucy Mangan, "The Crown review - the $£ 100 \mathrm{~m}$ gamble on the Queen pays off royally ", The Guardian (Royaume-Uni), 4 novembre 2016, consulté le 20 février 2019: https:// www.theguardian.com/tv-and-radio/2016/nov/04/the-crown-review-netflix-100m-gamble-onthe-queen-pays-off-royally.

18. Loughrey, op. cit.

19. Ibid.

20. Moreno, op. cit.

21. François Jost, «Fiction historique et réalisme », entretien publié par le Conseil Supérieur de l'audiovisuel, consulté le 20 février 2019: https://clesdelaudiovisuel.fr/Analyser/Medias-etrealite/Fiction-historique-et-realisme.

22. Omar Rincón, «Narcos da risa /El otro lado », El Tiempo (Colombie), 6 septembre 2015, consulté le 20 février 2019 : https://www.eltiempo.com/archivo/documento/CMS-16346263.

23. "Presidente santos se reune con Netflix », op. cit.

24. Entretien de Pedro Pascal dans CBS This Morning, YouTube, 2 septembre 2016, consulté le 20 février 2019 : https://www.youtube.com/watch?v=HC5G0bsSQtw. 
25. Joey Keeton, "Why the bad first season of 'Narcos' is worth watching », The Daily Dot (ÉtatsUnis), 5 septembre 2015, consulté le 20 février 2019: https://www.dailydot.com/upstream/ narcos-netflix-original-first-season-review/.

26. Faure et Taïeb, op. cit.

27. Rodrigo Amarante, « Tuyo ", Narcos (A Netflix Original Series), Lakeshore Records, 2015, 1'29.

28. Sibylla Brodzinsky, "Narcos is a hit for Netflix but iffy accents grate on Colombian ears ", The Guardian (Royaume-Uni), 17 septembre 2015, consulté le 20 février 2019: https:// www.theguardian.com/world/2015/sep/17/narcos-netflix-colombian-accents.

29. Équivalent $\mathrm{du}$ site Allociné au Brésil, consulté le 20 février 2019: http:// www.adorocinema.com/filmes/filme-133548/bilheterias/.

30. Moreno, op. cit.

31. Entretien de Wagner Moura dans The Tonight Show Starring Jimmy Fallon, Youtube, 9 janvier 2016, consulté le 20 février 2019 : https://www.youtube.com/watch?v=WwRdjf4eEgk.

32. Ibid.

33. "Yo ya no era Andrés, sino Pablo Escobar », Semana (Colombie), 11 janvier 2014, consulté le 20 février 2019 : http://www.semana.com/nacion/articulo/andres-parra-yo-ya-no-era-andres-sinopablo-escobar/407663-3.

34. David Musto, The American Disease: Origins of Narcotic Control, Oxfrod, Oxford University Press, 1999 ; William McAllister, Drug diplomacy in the twentieth century: An international history. Londres/ New York, Oxford University Press, 2000.

35. Conférence de presse de Wagner Moura et Pedro Pascal transmise par Reporte Indigo, Youtube, 17 août 2016, consulté le 20 février 2019 : https://www.youtube.com/watch?v=dD6Tr4LBCHQ.

36. D'après Jean-Paul Sartre, la différence entre le mensonge est la mauvaise foi est l'émetteur de l'action : alors que dans le mensonge le menteur trompe son interlocuteur, une personne de mauvaise foi se ment à elle-même. Jean-Paul Sartre, L'Etre et le néant, Paris, Gallimard, 1976.

37. Lucia Suarez Sang, «Ex-DEA agents who fought Pablo Escobar headline new NatGeo documentary ", Fox News (États-Unis), 30 août 2016, consulté le 20 février 2019: https:// www.foxnews.com/entertainment/ex-dea-agents-who-fought-pablo-escobar-headline-newnatgeo-documentary.

38. Entretien de Pedro Pascal avec Stephen Colbert, dans The Late Show, Youtube,30 août 2016, consulté le 20 février 2019 : https://www.youtube.com/watch?v=qe--y0apjcU.

39. Luis Chaparro, "Los agentes de la DEA que capturaron a Pablo Escobar nos dicen cómo atrapar al Chapo », Vice (Amérique latine), 3 novembre 2015, consulté le 20 février 2019 : https:// www.vice.com/es_latam/article/pp5kdg/los-agentes-de-la-dea-que-capturaron-a-pablo-escobarnos-dicen-como-atrapar-al-chapo.

40. Voir à ce sujet les entretiens de Pedro Pascal, op. cit, ou les mots de Wagner Moura dans la conférence de presse, op. cit., où ils déclarent que la légalisation de cannabis est la solution aux narcotrafic.

41. Publicité « Making of Narcos » par Netflix, Youtube, 20 septembre 2016, consulté le 20 février 2019 : https://www.youtube.com/watch?v=14eEcfoPauo.

42. L'expression très connue d'Escobar « plata o plomo » (littéralement, « l'argent ou le plomb ») peut être traduite par l'expression française « la bourse ou la vie ». 


\section{RÉSUMÉS}

Narcos est un succès pour Netflix. Positionnée comme une des meilleures productions de la plateforme, d'après son chef de contenu Ted Sarandos, la série a réussi à ouvrir le marché hispanophone (et bien d'autres) grâce à l'utilisation de l'Histoire colombienne, si tragique que parfois « la réalité dépasse la fiction » (S01E01). Toutefois, la réception colombienne de Narcos est ambiguë. À l'intérieur du pays, les critiques sont fortes et son succès très relatif: tant le gouvernement, comme le fils de Pablo Escobar, ainsi que les spécialistes de cinéma, questionnent l'image de puissance que la série véhicule du monde du trafic illicite de stupéfiants et notamment de cet anti-héros qu'est Pablo Escobar. Partant d'une réflexion sur l'utilisation de l'Histoire dans les produits culturels de masse, nous défendons l'idée que Narcos échoue à séduire les Colombiens, dans ses deux premières saisons, non pas par sa volonté de relater l'histoire colombienne, mais par la manière dont elle raconte l'Histoire.

Narcos is a success for Netflix. Positioned as one of the best productions on the platform, according to its content manager Ted Sarandos, the series succeeded in opening up the Spanishspeaking market (and many others) thanks to the use of Colombian History, so tragic that sometimes "reality exceeds fiction" (S01E01). However, the Colombian reception of Narcos is ambiguous. Inside the country, the critics are strong and its success very relative: both the government, like the son of Pablo Escobar, as well as the cinema specialists, question the image of power that the series conveys from the world of illicit traffic of narcotics and in particular of this anti-hero that is Pablo Escobar. Starting from a reflection on the use of History in mass cultural products, we defend the idea that Narcos fails to seduce Colombians, in its first two seasons, not by its desire to tell Colombian history, but by the way it narrates History.

\section{INDEX}

Mots-clés : Narcos, Netflix, Colombie, drogue, narration

Keywords : Narcos, Netflix, Colombia, drug, narrative

\section{AUTEUR}

\section{LUIS RIVERA-VÉLEZ}

Luis Rivera-Velez est doctorant au Centre d'études internationales (CERI) de Sciences Po Paris. Il étudie les politiques du cannabis en Amérique latine, en particulier le processus de mise à l'agenda de la légalisation en Uruguay, Colombie et Mexique entre 2010 et 2018. Il s'intéresse également aux lois sur l'avortement et à l'expansion des droits LGBTQ+ en Amérique latine.

Luis Rivera-Velez is a Ph.D. candidate at the Center of International Studies (CERI) in Sciences Po Paris. He studies the cannabis policies in Latin America, especially the process of agenda-setting of the cannabis laws in Uruguay, Mexico and Colombia between 2010 and 2018. He also studies the laws of abortion and the expansion of LGBTQ+ rights in Latin America. 